\title{
A Robust Solution Procedure for the Transonic Small-Disturbance Equation in Fluid-Structure Interactions
}

\author{
T.M. van Opstal ${ }^{*}, 1$, S.J. Hulshoff ${ }^{2}$ and C.V. Verhoosel ${ }^{3}$ \\ ${ }^{I}$ Faculty of Mechanical Engineering, Eindhoven University of Technology, P.O. Box 513, 5600 MB Eindhoven, The \\ Netherlands \\ ${ }^{2}$ Faculty of Aerospace Engineering, Delft University of Technology, Kluyverweg 1, 2629 HS Delft, The Netherlands \\ ${ }^{3}$ Institute for Computational Engineering \& Sciences, The University of Texas at Austin, 1 University Station C0200 \\ Austin, Texas 78712, USA
}

\begin{abstract}
A simple and robust discretization of the Low Frequency Transonic Small Disturbance (LF-TSD) equation is presented, suitable for applications such as fluid-structure interaction (FSI). Choices in the derivation and discretization of the LF-TSD equation are justified and the performance of the scheme is demonstrated for a typical nonlinear FSI problem.
\end{abstract}

Keywords: Fluid-structure interaction, transonic small-disturbance equation, finite differences, panel problem, divergence, flutter, limit cycle oscillation.

\section{INTRODUCTION}

Simplifications of the Navier-Stokes equations are employed in various fields. In early design stages quick computations are often used to give coarse performance indications in order to compare design options. In optimization problems, compact models are required for computational efficiency. In research on new numerical techniques, simplified models make numerical results easier to interpret. They also are desirable when large computing resources are required to establish convergence characteristics. A good example is the development of stochastic methods, where the Monte Carlo method is inevitably used for verification purposes.

In this paper an unsteady, nonlinear flow model, the LFTSD potential equation, is considered. Despite its simple form, it is able to capture nonlinear effects such as shock waves and long time lags. The nonlinearity allows for proper description of the sub- and supersonic regions typically coexisting in transonic flow. On the other hand, rotational, viscous and thermal effects are among those not captured. This may result in an acceptable loss of accuracy or even advantageous loss of complexity, depending on the problem at hand. Here, the LF-TSD flow description is applied to a prototypical unsteady FSI problem, viz. the one-dimensional panel. The low-frequency assumption is adopted, as it has proven to be appropriate for this class of problems [1,2].

Correct treatment of the changing type of PDE within the flow domain was a challenge to the CFD community until the early 70's, when Murman and Cole discovered a stable finite diffrence (FD) scheme for the steady Transonic

*Address correspondence to this author at the Faculty of Mechanical Engineering, Eindhoven University of Technology, P.O. Box 513, 5600 MB Eindhoven, The Netherlands; Tel: +31 (0)40 247 2271; Fax: +31 (0)40 247 5399; E-mail: t.m.v.opstal@tue.nl
Small Disturbance (TSD) equation [3, 4]. Unsteady formulations were the next hurdle. Explicit time-marching schemes were found to impose severe restrictions on the time step due to stability. Implicit schemes were thus adopted, using successive line over-relaxation [5], alternating-direction implicit methods [1] and approximate factorization [6] techniques. These show increasingly superior convergence rates, but employ segregated solution sweeps which require the correct choice of relaxation parameters to ensure numerical stability. Additionally, the stability of these algorithms is dependent on the discretization of the nonlinear terms. Unfortunately, the details of nonlinear term discretizations are not always discussed in the literature.

In view of these shortcomings, this paper aspires to describe and verify a robust discretization of the LF-TSD equation. First, the second-order time derivative is retained in the formulation, in order to exploit its known stabilizing effect [7]. Next, the discretization is based on the conservative form used in [8] rather than the more common forms found in [4, 5]. Finally, a fully monolithic time-marching approach is used. The latter provides convergence over a wide range of conditions, without the need to determine appropriate relaxation parameters. This can save considerable time when dealing with new problems.

This paper is organized as follows. In section 2 the derivation of the employed LF-TSD equation is presented. Subsequently, in 3 the discretization is described. Numerical verification of the presented model is performed in 4, followed by conclusions in 5 . The appendices contain detailed definitions of the scheme coefficients, and a proof that the current formulation is discretely conservative in space-time.

\section{ANALYTIC DESCRIPTION}

\subsection{Transonic Small Disturbance Equation}

The starting point for the derivation of the TSD equation is the unsteady Full Potential (FP) equation, which can be 
derived from the Euler equations assuming adiabatic irrotational flow $[9,10]$ :

$$
\begin{aligned}
& \phi_{t t}+2 u \phi_{x t}+2 v \phi_{y t}= \\
& \quad\left(a^{2}-u^{2}\right) \phi_{x x}-2 u v \phi_{x y}+\left(a^{2}-v^{2}\right) \phi_{y y},
\end{aligned}
$$

where the velocity is described by a potential through $(u, v)=\nabla \phi, a$ is the local sound speed and subscripts $t, x$ and $y$ denote temporal and spatial derivatives. With the assumption of isentropic flow, the energy equation can be written:

$\phi_{t}+\frac{u^{2}+v^{2}}{2}+\frac{a^{2}}{\gamma-1}=\frac{u_{\infty}^{2}}{2}+\frac{a_{\infty}^{2}}{\gamma-1}$.

Considerable simplification of the above system can be made by assuming a uniform flow with small disturbances superimposed on it. Thin airfoils and panels with small displacements (which will be treated in section 4) are typical applications for which this assumption is appropriate. In this context, assuming only small disturbances has the further benefit of eliminating the need for fluid mesh deformation to accommodate changes in the solid boundary geometry. The disturbance potential, $\varphi$, is defined by

$\phi=u_{\infty}(x+\varphi)$, with $\varphi_{x}, \varphi_{y} \ll 1$

and $\infty$ indicating freestream conditions. $\varphi$ is included within the brackets and therefore scaled with $u_{\infty}$. This is important for maintaining robustness over a range of conditions. ${ }^{1}$

It has been observed that low-frequency components of the FP equation normally dominate the nonlinear response [1]. Thus the following assumption is made:

$\varphi_{t} \ll \varphi_{\alpha}, \alpha=x, y$.

By inserting the above into the energy equation and ignoring higher-order terms in $x$ and $y$, the speed of sound $a$ can be approximated by

$a^{2} \approx a_{\infty}^{2}-(\gamma-1) u_{\infty}^{2} \varphi_{x}-(\gamma-1) u_{\infty} \varphi_{t}$.

Equation 1 is then linearized, retaining only the nonlinear term with $\varphi_{x} \varphi_{x x}$ from the first right-hand side term as it is known to be relatively large near shocks. This implies that no account is made of shock components in the vertical direction [11]. The first term in (1) is kept as it has a known stabilizing effect [7].

Finally, the equation is brought into nondimensional form. To this end $t$ is scaled by $l / a_{\infty}$ and $x, y$ and $\varphi$ by $l$, where $l$ is a characteristic length. The LF-TSD equation then becomes

$\varphi_{t t}+2 M_{\infty} \varphi_{x t}=A \varphi_{x x}+\varphi_{y y}$

\footnotetext{
${ }^{1}$ An alternative choice for the potential would be $\phi=u_{\infty} x+\varphi$. When this is nondimensionalized, the nonlinear term will scale with $M_{\infty}$ instead of with $M_{\infty}^{2}$ which turns out to postpone shock formation in the supersonic regime.
}

where $M_{\infty}$ is the freestream Mach number and $A$ is a nonconstant coefficient

$$
A(\varphi):=1-M_{\infty}^{2}-(\gamma+1) M_{\infty}^{2} \varphi_{x},
$$

making the equation nonlinear. Solving for $\varphi$ determines the complete solution. The velocity field $(u, v)$ is given through the definition of the potential in (3). Density, temperature and pressure can be found by using isentropic relations. The linearized pressure in nondimensional form is

$\bar{p}:=\frac{p-p_{\infty}}{\rho_{\infty} a_{\infty}^{2}} \approx \frac{1}{\gamma}-M_{\infty} \varphi_{t}-M_{\infty}^{2} \varphi_{x}$.

In the sequel this relation will be employed as a coupling mechanism in the FSI problem.

\subsection{Characteristics of the LF-TSD Equation}

The characteristics of equation (5) have implications for the way in which it should be discretized. Indeed, early discretizations of the TSD equation failed to take this into account, yielding unresolvable instabilities in transonic flow [11]. Before outlining the behaviour of the characteristics, the coefficient $A$ from equation (6) is examined.

The sign of $A$ determines the type of the PDE, such that the latter changes from elliptic to hyperbolic type in subsonic and supersonic regions of the solution respectively. This can be seen from the following analysis in a sonic point of the flow:

$$
0=\frac{a^{2}-\left(u^{2}+v^{2}\right)}{a_{\infty}^{2}} \approx 1-M_{\infty}^{2}-(\gamma+1) M_{\infty}^{2} \varphi_{x}=A .
$$

For clarity the analysis is performed taking two dimensions at a time, as in [5], see also [1,11]. Equation (5) is locally linearized by taking $A(\varphi)$ to be constant.

\section{a. Characteristic Velocities}

The velocity of disturbances in streamwise direction can be found by substituting the solution $\varphi=f(\alpha t-x)$ into (5). This gives

$$
\left(-\alpha^{2}+2 M_{\infty} \alpha+A\right) f^{\prime \prime}=0 \Rightarrow
$$

$\left.\frac{d x}{d t}\right|_{\text {char }}=\alpha=M_{\infty} \pm \sqrt{M_{\infty}^{2}+A}$

Causality dictates that information only travels in time in the positive sense. Thus, information can only travel along the characteristic in one of the two directions (save the sonic case $d x / d t=0)$. In contrast to the analysis in [5], information travels downstream with a finite velocity due to the presence of the $\varphi_{t t}$ term. The receding characteristic has a negative slope in locally subsonic flow, i.e. information travels upstream with a finite velocity. For supersonic conditions, the characteristic has a positive slope and information ceases to travel upstream, properly reflecting the physics of the flow.

\section{b. Characteristic Directions}

To determine the characteristic directions, the steady state solution $\varphi=g(\beta x-y)$ is inserted. The TSD equation becomes 


$$
\begin{gathered}
\left(A \beta^{2}+1\right) g^{\prime \prime}=0 \Rightarrow \\
\left.\frac{d y}{d x}\right|_{\text {char }}=\beta=\frac{ \pm 1}{\sqrt{-A}} .
\end{gathered}
$$

For subsonic flows $(A>0)$ the gradient becomes undefined and information is carried through the domain in every direction. For supersonic flows information travels along the characteristics solely in positive $x$-direction. Thus characteristics with a positive slope emanate from the bottom of the domain and a negative slope from the top of the domain.

\subsection{Boundary Conditions}

In this paper the panel problem is considered, for which the flow domain is bounded by a solid surface at the bottom, and extends to infinity in the other three directions. Farfield conditions will be used to truncate the domain such that numerical is found on a finite, rectangular domain $\Omega$ with boundary $\partial \Omega$. This boundary is a disjoint union of the segments $\partial \Omega_{\mathrm{wl}}$ (wall), $\partial \Omega_{\mathrm{if}}$ (inflow), $\partial \Omega_{\text {lat }}$ (lateral) and $\partial \Omega_{\text {of }}$ (outflow) as shown in Fig. (1). Boundary conditions (bcs) are to be defined on the entirety of $\partial \Omega$. For bcs of the form $\forall x \in \partial \Omega_{\text {seg }}: f(\varphi)=g(x)$ the short notation $f\left(\left.\varphi\right|_{\text {seg }}\right)=g(x)$ is adopted for each of the respective boundary segments 'seg'.

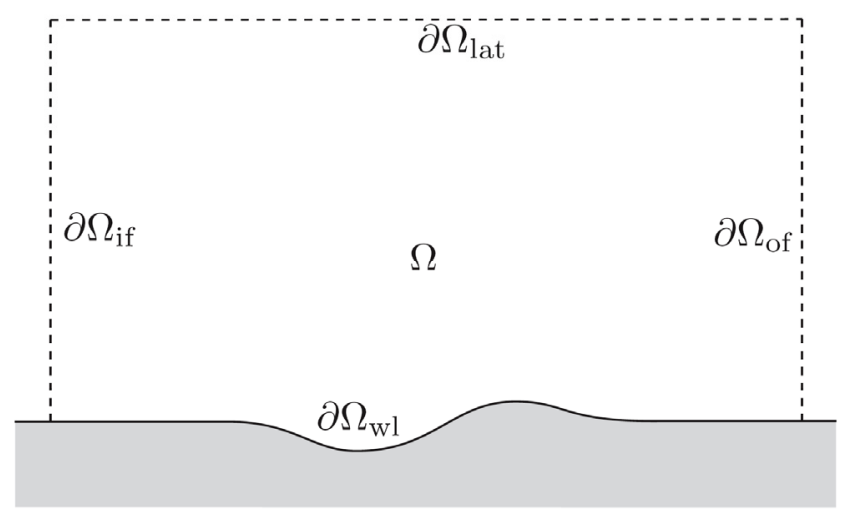

Fig. (1). Flow domain $\Omega$ and its boundaries.

\section{a. Transpiration Boundary}

The flow is unable to penetrate the wall. If the contour of the body is described by $y_{\mathrm{wl}}(x)$, applying the small disturbance assumption (3), the transpiration bc becomes (see for instance [7, p. 45]):

$\left.\frac{v}{u}\right|_{\mathrm{wl}}=\frac{d y_{\mathrm{wl}}}{d x} \Rightarrow \varphi_{y} \mathrm{I}_{\mathrm{wl}}=\left(1+\varphi_{x}\right) \frac{d y_{\mathrm{wl}}}{d x} \approx \frac{d y_{\mathrm{wl}}}{d x}$.

\section{b. Subsonic Farfield}

The farfield boundaries are to be defined sufficiently far away from the region of interest such that disturbances are sufficiently small. The disturbance component orthogonal to the boundary is taken:

$\varphi_{x} \mathrm{I}_{\text {if }}=\varphi_{x} \mathrm{I}_{\text {of }}=\varphi_{y} \mathrm{I}_{\mathrm{lat}}=0$.
This way after discretization, $\varphi$ on the boundary can always be related to the internal solution (see section 3.2 for details).

\section{c. Supersonic Farfield}

Based on the discussion of characteristic directions, the inflow condition is simply $\varphi \mathrm{l}_{\text {if }}=0$, while the outflow is completely determined by the internal equations. For the calculations presented here, $\left.\varphi_{y}\right|_{\text {lat }}=0$ was used for the lateral boundary. This is a reflecting condition, but for the supersonic cases considered, the lateral boundary was set far enough away so that the reflections could not influence the panel region. More sophisticated approaches for lateral boundaries can be found in [8] and [12].

\section{DISCRETIZATION}

In transonic conditions, different regions in the flow will have different domains of dependence. If these domains are not treated properly, the resulting solutions may contain nonphysical expansion shocks, or might not be found at all [8]. Murman and Cole [13] were the first to select an appropriate domain of dependence using a switching operator. The discretization presented here is a mass conserving, unsteady variation of this original FD scheme.

\subsection{Spatial Disctretization}

The flow field is approximated at nodes arranged on a Cartesian stretched grid $\Omega_{h}:=\left\{\left(x_{i}, y_{j}\right) \mid i \in\{1, \ldots, H\} \wedge j \in\{1, \ldots, V\}\right\}$. Values at these nodes will be denoted $\varphi_{i, j}:=\varphi\left(x_{i}, y_{j}\right)$.

Following [15], the conservation form of the LF-TSD equation (5) is used. It is given by

$U_{t}+P_{x}+Q_{y}=0$

with

$U:=-\varphi_{t}-2 M_{\infty} \varphi_{x}$,

$P:=\left(1-M_{\infty}^{2}\right) \varphi_{x}-\frac{\gamma+1}{2} M_{\infty}^{2} \varphi_{x}^{2}$,

$Q:=\varphi_{y}$

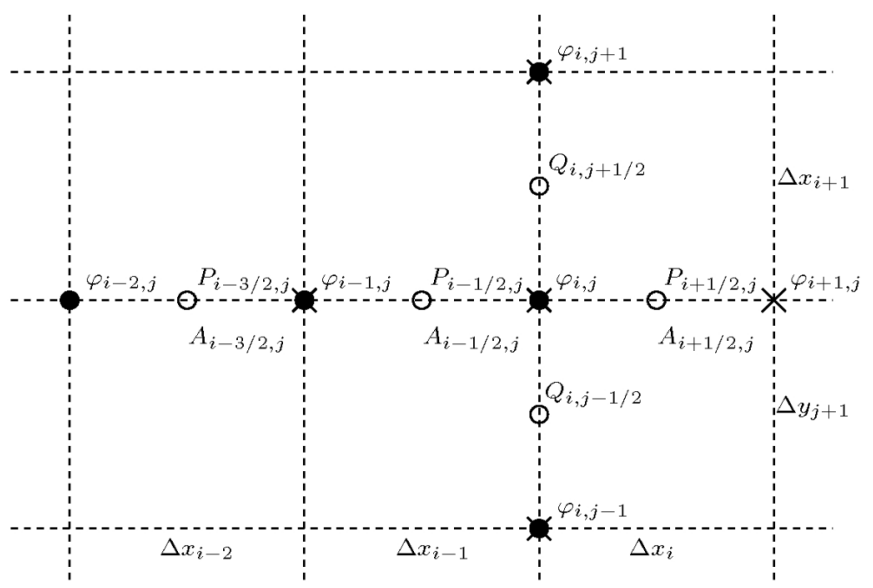

Fig. (2). The two stencils used: an upwind stencil depicted in solid dots and a central stencil in x-marks. The open circles mark the half-points. 
The values of $P$ are taken at half-points horizontally and $Q$ at half-points vertically to arrive at a compact stencil, see Fig. (2). This is because the derivatives of $\varphi$ can be determined to second-order accuracy using the two immediate neighbours. Next, the equation can be evaluated at every internal node using central differencing for $P_{x}$ and $Q_{y}$. For example, for the central differencing of $P_{x}$ the discrete derivative is

$\left.P_{x}\right|_{i, j} ^{\text {centr. }}=\frac{P_{i+1 / 2, j}-P_{i-1 / 2, j}}{1 / 2\left(\Delta x_{i-1}+\Delta x_{i}\right)}$.

In supersonic regions an upwinding scheme is used to evaluate $P_{x}$, to reflect the physical domain of dependence. This is done by moving the $x$-derivative stencil one node downstream. Based on Murman's mass conserving approach [12] the first-order accurate scheme

$\dot{U}_{i, j}^{n+1}+2\left(1-\mu_{i, j}\right) \frac{P_{i+1 / 2, j}^{n+1}-P_{i-1 / 2, j}^{n+1}}{\Delta x_{i-1}+\Delta x_{i}}+$

$+2 \mu_{i-1, j} \frac{P_{i-1 / 2, j}^{n+1}-P_{i-3 / 2, j}^{n+1}}{\Delta x_{i-1}+\Delta x_{i}}+2 \frac{Q_{i, j+1 / 2}^{n+1}-Q_{i, j-1 / 2}^{n+1}}{\Delta y_{j-1}+\Delta y_{j}}=0$

is proposed by Sankar [8], see Fig. (2). The right superscript $n+1$ denotes the next time level. Note here that the denominator of the upwind term is not $\Delta x_{i-2}+\Delta x_{i-1}$. This choice has been made to guarantee conservation (see appendix A). For mildly stretched grids the loss of accuracy is small. The switching operator $\mu$ distinguishes between the sub- and supersonic schemes and is defined as

$\mu_{i, j}=\operatorname{sgn}\left(A_{i, j}-\left|A_{i, j}\right|\right)$

with

$$
A_{i, j}=\left(1-M_{\infty}^{2}\right)-(\gamma+1) M_{\infty}^{2} \varphi_{x} l_{i, j}
$$

and $\operatorname{sgn}(\alpha):=1$ if $\alpha \leq 0$ and 0 otherwise. Note that in every internal node the terms $U_{t}$ and $Q_{y}$ are taken into consideration. For $P_{x}$ four different types of nodes can be distinguished:

1. Subsonic point Both $\mu_{i, j}$ and $\mu_{i-1, j}$ are 0 and $P_{x}$ is discretized by central differencing, reflecting the local elliptic type of the equation.

2. Supersonic point Both $\mu_{i, j}$ and $\mu_{i-1, j}$ are 1 and $P_{x}$ is found through upwind differencing, reflecting the fact that information can only travel downstream in supersonic flow.

3. Parabolic point Now $\mu_{i, j}=1$ whilst $\mu_{i-1, j}=0$. The discretized equation reduces to $U_{t}+Q_{y}=0$. Using linear analysis, Murman [4] demonstrates that the central nor the upwind discretization of $P_{x}$ is stable in a parabolic point.
4. Shock-point At a shock $\mu_{i, j}=0$ whilst $\mu_{i-1, j}=1$. Both the central and upwind $P_{x}$ contribute in this point. Using this operator in the shock point guarantees that the weak solution (which is the shock discontinuity) is found $[4,11]$.

An important feature of this scheme is the fact that it is conservative. Typically conservative methods produce shocks which are stronger and further downstream than those of nonconservative methods. Spuriously the non-conservative solution is often closer to the Navier-Stokes solution, where viscosity is the mechanism responsible for a weaker shock located further upstream [8]. The conservative scheme is chosen here to ensure that the numerical solution approaches the theoretical jump conditions [4]. Conservation is demonstrated in appendix A using Gauss' Theorem.

The next step is the discretization of $\dot{U}, P$ and $Q$ at the half-points as required by (13). The nonlinear $P$ is first linearized with respect to $\varphi_{x}$;

$U_{i, j}^{n+1}=-\left.\dot{\varphi}\right|_{i, j} ^{n+1}-\left.2 M_{\infty} \varphi_{x}\right|_{i, j} ^{n+1}$,

$P_{i+1 / 2, j}^{n+1} \approx P_{i+1 / 2, j}^{n}+\left.\left(\left.\varphi_{x}\right|_{i+1 / 2, j} ^{n+1}-\left.\varphi_{x}\right|_{i+1 / 2, j} ^{n}\right) \frac{d P}{d \phi_{x}}\right|_{i+1 / 2, j} ^{n}$

$\left.\approx A_{i+1 / 2, j}^{n} \varphi_{x}\right|_{i+1 / 2, j} ^{n+1}+\frac{\gamma+1}{2} M_{\infty}^{2}\left(\frac{\varphi_{i+1, j}^{n}-\varphi_{i, j}^{n}}{\Delta x_{i}}\right)^{2}$,

$Q_{i, j+1 / 2}^{n+1}=\left.\varphi_{y}\right|_{i, j+1 / 2} ^{n+1} \approx \frac{\varphi_{i, j+1}^{n+1}-\varphi_{i, j}^{n+1}}{\Delta y_{j}}$.

Substituting equations (15) into (13) yields the discretized equation for every internal node $(x, y) \in \Omega_{h} \backslash \partial \Omega$. Collecting these equations into a banded matrix, it is convenient to use an index $k$ defined through the bijection $k:(i, j) \mapsto k, k=i+(j-1) H$. Now the single PDE (13) can be written as the system

${ }^{0} M_{k} \ddot{\varphi}_{k}^{n+1}+\sum_{l \in L}^{l} C_{k} \dot{\varphi}_{k+l}^{n+1}+\sum_{m \in M}^{m} K_{k}^{\overline{n+1}} \varphi_{k+m}^{n+1}=r_{k}^{\overline{n+1}}$

with $L:=\{-1,0\}, M:=\{-H,-2,-1,0,1, H\}$ and diagonals given in appendix $\mathrm{B}$. As the time-marching scheme will be implicit, the bar denotes the intermediate solution of the potential field, which is iterated until converged. In these equations the nonlinear coefficient $A$ is defined as

$A_{i+1 / 2, j}^{\overline{n+1}}:=1-M_{\infty}^{2}-(\gamma+1) M_{\infty}^{2} \frac{\varphi_{i+1, j}^{\overline{n+1}}-\varphi_{i, j}^{\overline{n+1}}}{\Delta x_{i}}$,

and the switching operator $\mu$ as

$$
\mu_{i, j}^{\overline{n+1}}:=\operatorname{sgn}\left(A_{i, j}^{\overline{n+1}}-\left|A_{i, j}^{\overline{n+1}}\right|\right)
$$

with 
$A_{i, j}^{\overline{n+1}}:=1-M_{\infty}^{2}-(\gamma+1) M_{\infty}^{2} \frac{\varphi_{i+1, j}^{\overline{n+1}}-\varphi_{i-1, j}^{\overline{n+1}}}{\Delta x_{i-1}+\Delta x_{i}}$.

Note that $\varphi_{x}$ is evaluated in the half-points in (17), but at the nodes in (18) using the respective central difference schemes. The calculation of $A$ at the half-points arises from the use of the conservative form (12), distinguishing this scheme from for instance [4]. The central discretization of $\mu$ is crucial for the proper functioning of the method.

\subsection{Boundary Conditions}

What remains is to discretize the equations for the boundary nodes $(x, y) \in \Omega_{h} \cap \partial \Omega$. By introduction of virtual points, the fluid bc's from section 2.3 may be written as

\section{a. Transpiration Boundary}

$\frac{\varphi_{i, 2}^{n+1}-\varphi_{i, 0}^{n+1}}{2 \Delta y_{1}}=\left.\frac{d y_{\mathrm{wl}}}{d x}\right|_{i}$

\section{b. Subsonic Farfield}

$\frac{\varphi_{2, j}^{n+1}-\varphi_{0, j}^{n+1}}{2 \Delta x_{1}}=0$

$\frac{\varphi_{i, V+1}^{n+1}-\varphi_{i, V-1}^{n+1}}{2 \Delta y_{V-1}}=0$

$\frac{\varphi_{H+1, j}^{n+1}-\varphi_{H-1, j}^{n+1}}{2 \Delta x_{H-1}}=0$

\section{c. Supersonic Farfield}

$\varphi_{-1, j}^{n+1}=\varphi_{0, j}^{n+1}=0$

By constructing the discrete fluid equations according to (16) and eliminating the virtual points using eqs. (19) and farfield values of $\mu$ where $i=0$, the matrix diagonals can be assembled to

$M^{\mathrm{ff}} \ddot{\varphi}^{n+1}+C^{\mathrm{ff}} \dot{\varphi}^{n+1}+K^{\mathrm{ff}, \overline{\mathrm{n}+1}} \varphi^{n+1}=r^{\mathrm{f}, \overline{\mathrm{n}+1}}$.

Stabilizing techniques such as flux limiters, domainsweeping and relaxation are used nor required to guarantee stable time integration. This feature distinguishes the present scheme from earlier work, such as [1, 2, 5-7].

\subsection{Time Integration}

To maximize robustness a first-order backward differencing scheme is used for time integration. Considering the second-order system

$M \ddot{x}^{n+1}+C x^{n+1}+K^{\bar{n}} x^{n+1}=r^{\bar{n}}$

the solution $x^{n+1}$ at the next time level $n+1$, is computed using the configurations from the last two time levels: $x^{n}$ and $x^{n-1}$. By Taylor series analysis difference operators can then be defined as

$D_{t}:=\frac{1}{\Delta t}\left[I d_{N},-I d_{N}, \varnothing_{N \times N}\right]$
$D_{t t}:=\frac{1}{\Delta t^{2}}\left[I d_{N},-2 I d_{N}, I d_{N}\right]$

acting on $\left\{x^{n+1} ; x^{n} ; x^{n-1}\right\}$, yielding the first-order Backward Euler differencing scheme [1, Ch. 2]

$$
\begin{aligned}
x^{n+1}= & \left(\frac{M}{\Delta t^{2}}+\frac{C}{\Delta t}+K\right)^{-1} \times \\
& \left(r+\frac{M}{\Delta t^{2}}\left(2 x^{n}-x^{n-1}\right)+\frac{C}{\Delta t} x^{n}\right) .
\end{aligned}
$$

First-order accuracy is accepted in this context as accuracy requirements are outweighed by robustness in new applications.

\section{RESULTS}

\subsection{Biconvex Airfoil}

The transonic flow around a biconvex airfoil is a known test case for transonic flows. It features sharp leading and trailing edges and a supersonic pocket that is terminated by a shock. The circular-arc airfoil shape is approximated by

$y_{\mathrm{wl}}=2 t_{\mathrm{af}}(1-x) x$ on $x \in[0,1]$

with $t_{\mathrm{af}}$ the airfoil thickness.

The solution of the problem on a grid with $(x, y) \in[-5 ; 15] \times[0 ; 20], \Delta x=1 / 64$ and $10 \%$ exponential stretching away from the airfoil, is visualized in Fig. (2). The outflow boundary has been placed further away to avoid errors induced by shock reflections, though Table 1 shows that the positions of in- and outflow boundaries have only a minor effect on the solution. The pressure distribution over the airfoil is given in Fig. (2). Note that the compact stencil of Fig. (2) results in a limited smearing out of the shock over three mesh points.

Spatial convergence is studied by looking at $\varepsilon_{\varphi}$, the $\ell^{2}$ norm of the error with respect to the reference solution ( $\Delta x=1 / 256)$ at equilibrium. Equilibrium is defined as $\|\dot{\varphi}\|_{\infty} \leq 1 \times 10^{-8}$. The shock position converges to $x_{\mathrm{sh}}=0.737$. Spatial convergence is linear as expected because of the first-order spatial discretization, see Fig. (4).

\subsection{Linear Stability of a Flexible Panel}

In the next two test cases, the performance of the discretization in a fluid-structure interaction setting is demonstrated. The panel problem, as considered here, consists of a one-dimensional panel clamped at both ends and submersed in the two-dimensional fluid described above, see Fig. (5).

The panel displacement $w$ is described by the beam equation [14];

$w_{t t}+\lambda^{2} w_{x x x x}=\zeta(\bar{p}-\pi)$

given the ratio of time-scales $\lambda$, ratio of masses $\zeta$ and some pressure forcing $\pi$ : 


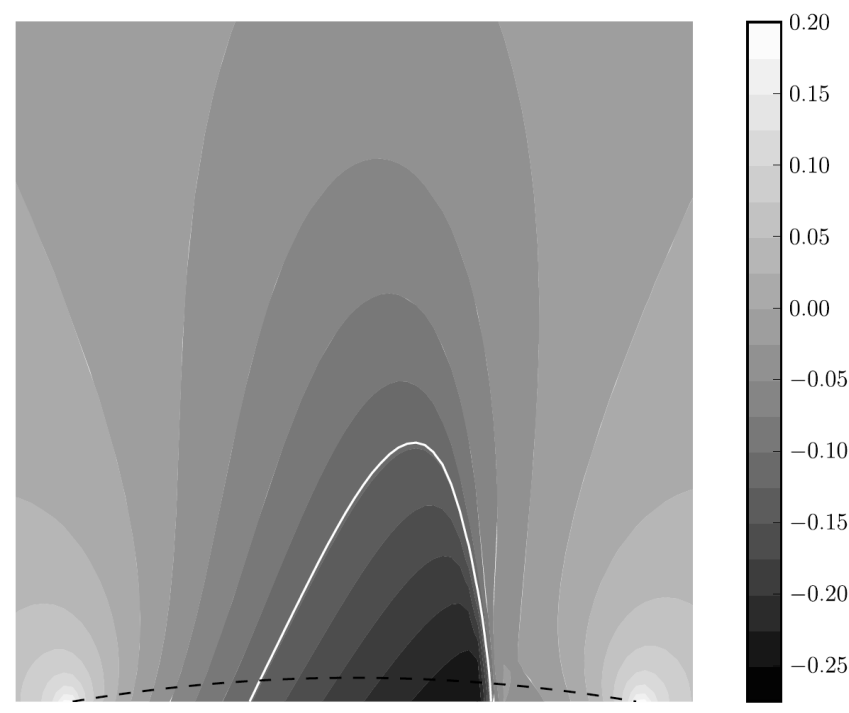

(a) Flow around a biconvex airfoil with isobars. The white contour indicates the position of the supersonic pocket. The airfoil contour is given to scale by the dashed black line. Due to the linearization of boundary conditions the computational domain is rectangular. The airfoil geometry is merely superimposed in this figure to give an indication of the local input of the structure to the TSD solution.

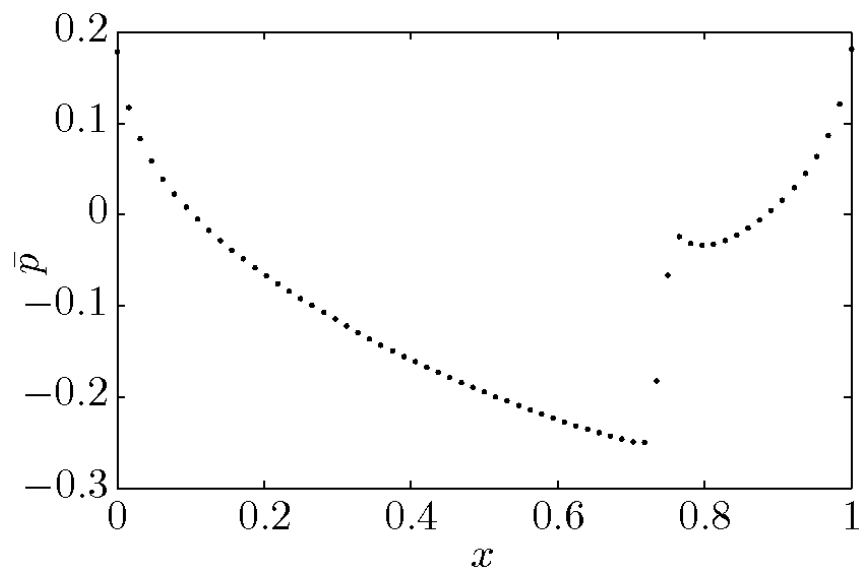

(b) Airfoil surface pressure distribution.

Fig. (3). Steady state solution for a biconvex airfoil with a thickness of $8.4 \%$ at $M_{\infty}=0.84$.

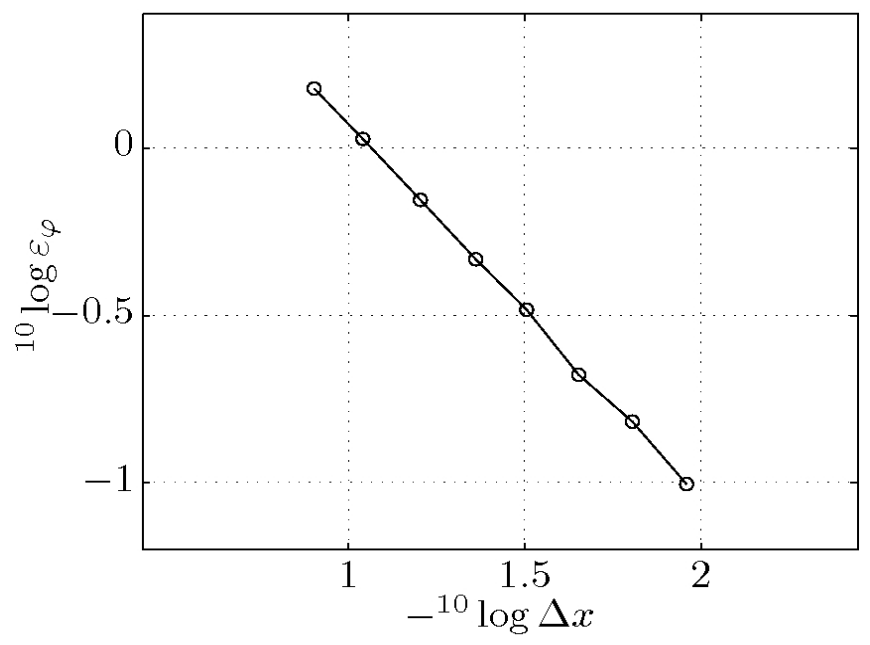

Fig. (4). Spatial convergence for the biconvex airfoil test case.
Table 1. Shock position $x_{\mathrm{sh}}$ as a function of proximity of inand outflow boundaries, $\min x$ and $\max x$ resp. with $\|\dot{\varphi}\|_{b_{0}} \leq 10^{-6}$ and a lateral boundary placed at $\max$ $\boldsymbol{y}=\mathbf{2 0}$.

\begin{tabular}{|c|c|c|}
\hline $\min \boldsymbol{x}$ & $\max \boldsymbol{x}$ & $\boldsymbol{x}_{\text {sh }}$ \\
\hline \hline-20 & 5 & 0.7379 \\
\hline-20 & 10 & 0.7371 \\
\hline-20 & 15 & 0.7370 \\
\hline-20 & 20 & 0.7370 \\
\hline-15 & 20 & 0.7370 \\
\hline-10 & 20 & 0.7370 \\
\hline-5 & 20 & 0.7374 \\
\hline
\end{tabular}

$\lambda=\frac{l a_{\infty}^{-1}}{\left(\rho^{\mathrm{s}} h\right)^{1 / 2} l^{2}(E I)^{-1 / 2}}$,

$\zeta=\frac{\rho_{\infty} l}{\rho^{\mathrm{s}} h}$,

$\pi=\frac{p_{\text {forcing }}-p_{\infty}}{\rho_{\infty} a_{\infty}^{2}}$,

where $\infty$ denotes freestream variables and structural parameters are clarified in Fig. (5). The fluid is coupled to structural response through the transpiration boundary condition by the material derivative:

$$
M_{\infty} \varphi_{y}=w_{t}+M_{\infty} w_{x} .
$$

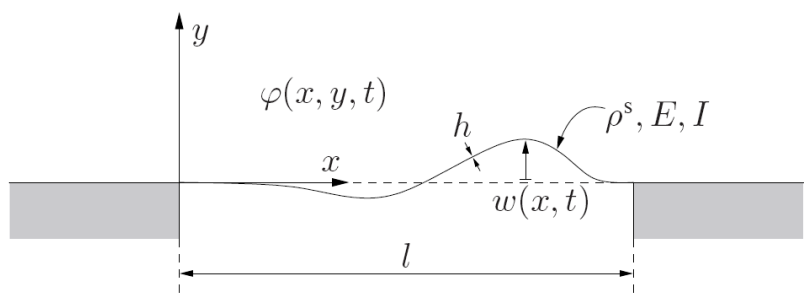

Fig. (5). Panel schematic with structural density $\rho^{\mathrm{s}}$, Young's modulus $E$ and sectional stiffness $I$.

The panel is divided into $n_{e}$ finite elements with nodes coinciding with fluid mesh points and third order Hermitian polynomials defined over them. These shape functions are chosen to ensure the $C^{1}$ continuity required in the weak form of (24). The pressure $\bar{p}$ is obtained from (7).

For the linear, deterministic panel stability problem analytical and numerical results [15] are available. Two phenomena can be distinguished: divergence, which is a static instability occurring at subsonic speeds; and flutter, a generally supersonic dynamic instability. The analysis presented here closely follows Verhoosel et al. [15], including the parameters used there, see Table 2. Computations were performed on the domain $(x, y) \in[-2 ; 3] \times[0 ; 3]$ with constant grid spacing. 
Table 2. Physical parameters for the linear divergence and flutter tests.

\begin{tabular}{|c|c|c|}
\hline & Divergence & Flutter \\
\hline \hline$\lambda^{2}$ & $4.204 \times 10^{-5}$ & $2.027 \times 10^{-4}$ \\
\hline$\zeta$ & $3.348 \times 10^{-1}$ & $7.513 \times 10^{-2}$ \\
\hline
\end{tabular}

\section{a. Divergence}

An estimate for the divergence Mach number can be obtained by

$K x=0$,

which only has a nontrivial solution when the matrix is singular. Thus, finding the divergence solution is identical to increasing the Mach number until the smallest magnitude eigenvalue becomes zero. The divergence Mach number is defined as the smallest Mach number at which equality holds. Kornecki et al. [16] proposes the semi-analytical solution

$M_{\mathrm{div}} \approx \sqrt{177 \lambda^{2} / \zeta}$

For the conditions considered, equation (26) yields $M_{\text {div }} \approx 0.1491$, a value of 0.149 was found in [15]. Numerical experiments yield the comfortably accurate $M_{\text {div }} \approx 0.1486$ for $n_{e}=128$. The mode shape and convergence behaviour are given in Fig. (6) and good correspondence is found with [15]. The error $\varepsilon_{M}$ is defined as the relative difference in $M_{\text {div }}$ with respect to the reference solution $n_{e}=128$.

\section{b. Flutter}

To find the flutter instability boundary a harmonic solution $\left(x=\sum_{i \leq N} v_{i} e^{\mu_{i} t}\right)$ is substituted into the linearized form of (21), yielding the quadratic eigenvalue problem

$\left[\mu_{i}^{2} M+\mu_{i} C\left(M_{\infty}\right)+K\left(M_{\infty}\right)\right] v_{i}=0$

with $N$ eigenvalues $\mu_{i} \in \mathbb{C}$ and eigenvectors $v_{i}$. The system is unstable when $\exists i: \operatorname{Re}\left[\mu_{i}\right]>0$ and the flutter Mach number is the smallest $M_{\infty}$ at which this occurs.

The flutter Mach number converges to 2.268 for $n_{e}=128$, which corresponds nicely with the result from [17] for these parameters; $M_{\mathrm{fl}}=2.27$. The mode shape and convergence behaviour are plotted in Fig. (7). Again, the Mach number and convergence behavior compare accurately with the results in [15].

\subsection{Limit Cycle Oscillation of a Flexible Panel}

As a third case the full unsteady, nonlinear, coupled model is considered: the transonic Lco. An LCO is a periodic motion described by a closed trajectory in phase space (see texts on dynamical systems such as [18]). In supersonic (a) Divergence mode shape.

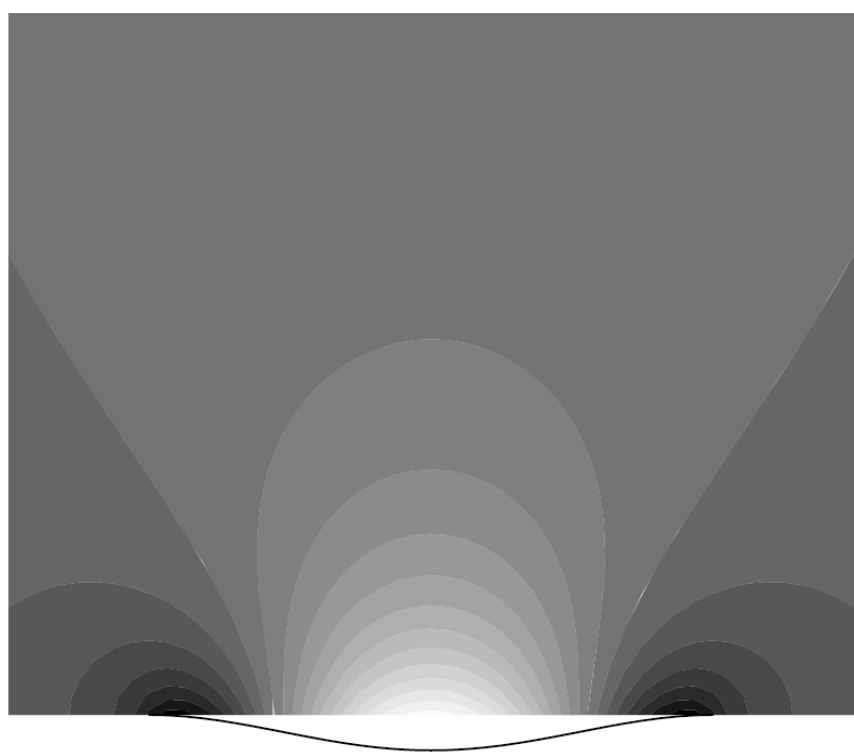

(b) Spatial convergence.

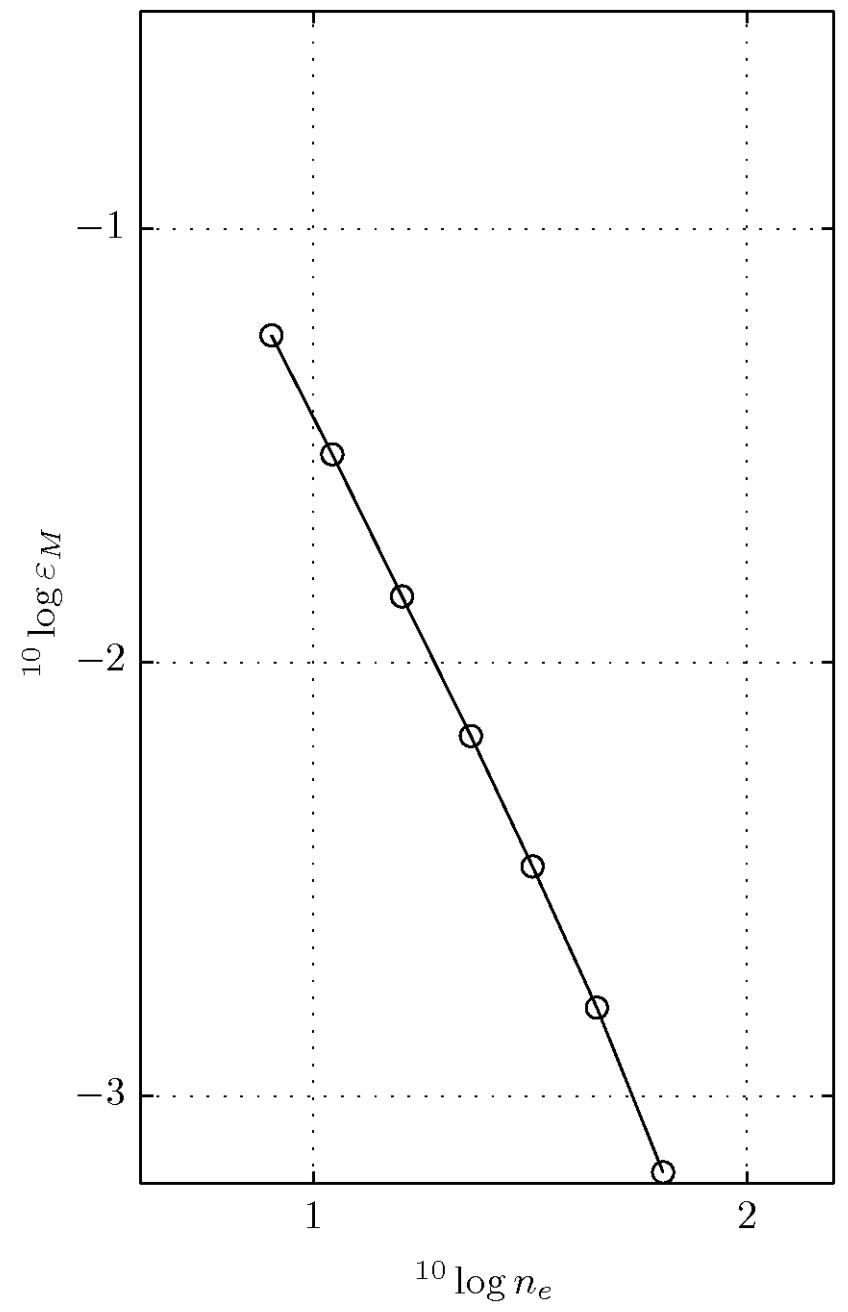

Fig. (6). Simulation summary for the linear divergence test case.

flow transient shock formation can prevent a flutter mode from growing unboundedly. In this case the response becomes periodic. For a typical LCO, Fig. (8) gives snapshots 
(a) Flutter mode shape.

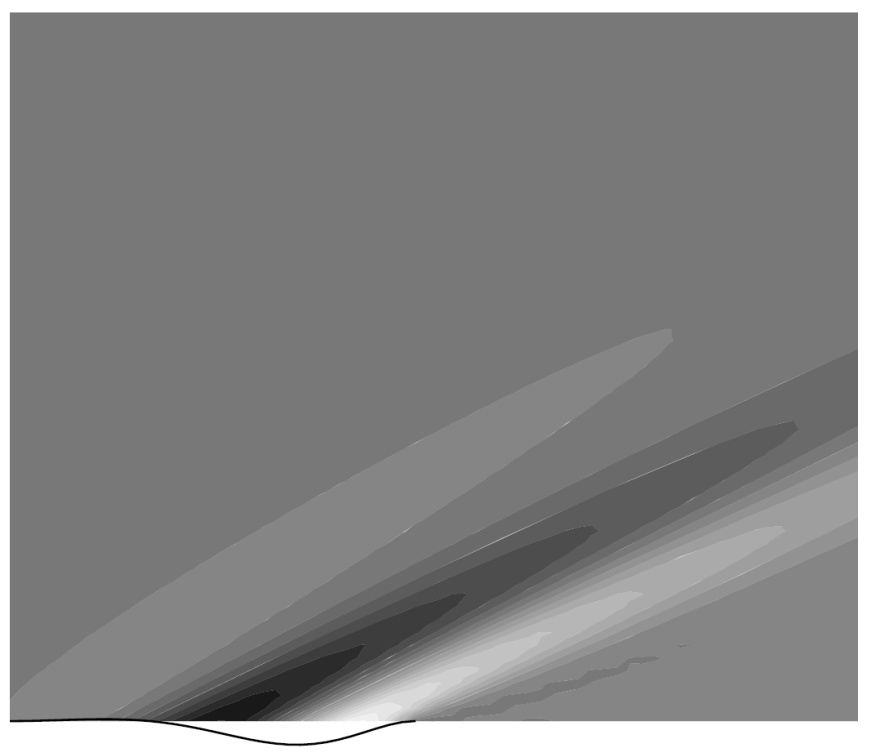

(b) Spatial Convergence.

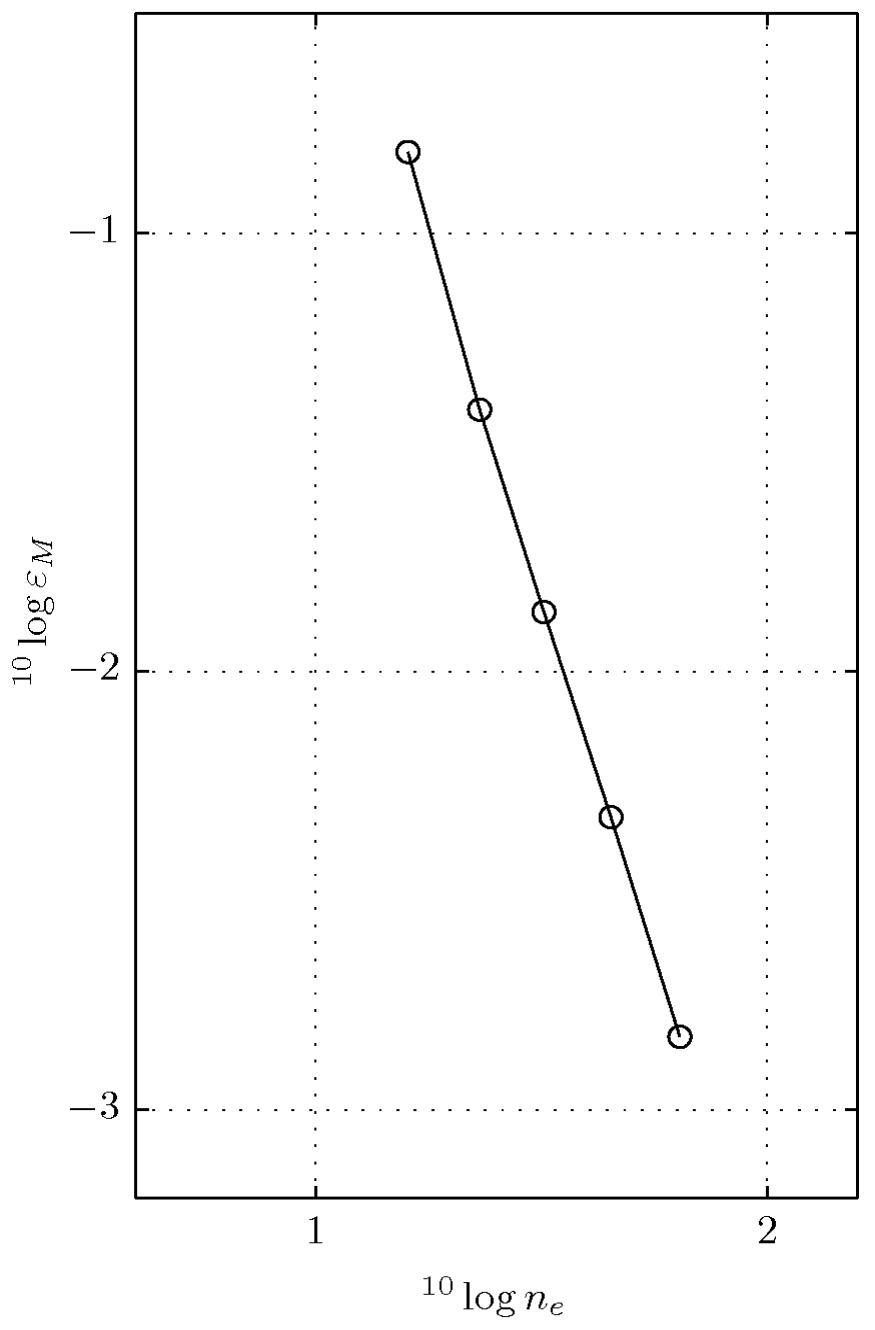

Fig. (7). Simulation summary for the linear flutter test case.

of the solution at different stages in one period. It is necessary to use time integration to find this solution, thereby making no a priori assumptions on the solution, because two large subsonic pockets disappear and reappear during one period. This solution can thus not be found by linearizing shock motion. The parameters used here are $M_{\infty}=1.20, \lambda^{2}=4.449 \times 10^{-4}$ and $\zeta=5.070 \times 10^{-2}$. $\mathrm{i}$

iii

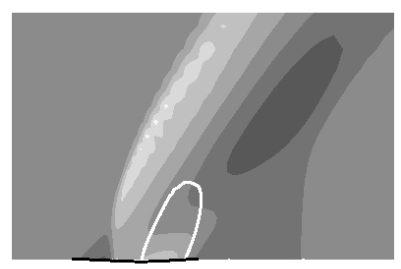

$\mathrm{V}$

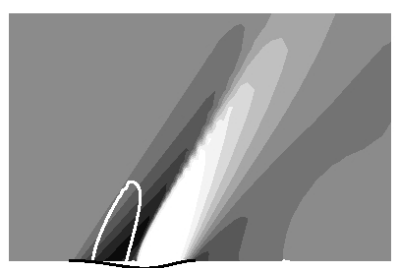

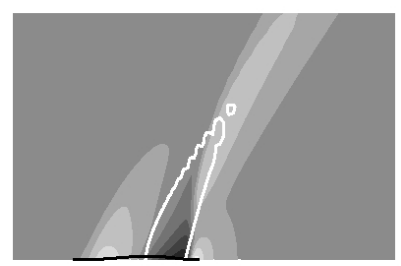

ii

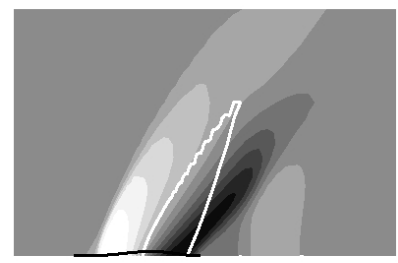

iv

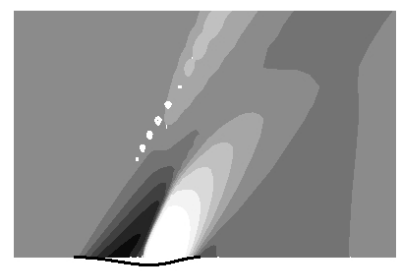

vi

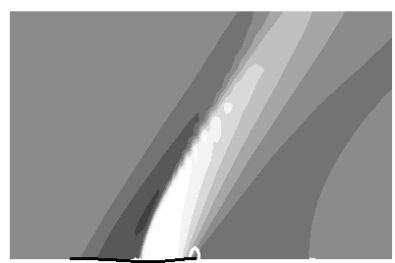

Fig. (8). Consecutive snapshots of the periodic LCO motion with isobars. White contours delimit subsonic bubbles, panel displacement is given in black.

This simulation did not require tuning of any relaxation parameters. Fig. (9) shows the convergence study performed for this case in the space-time energy norm defined by

$$
\begin{aligned}
\|w\|_{U} & :=\left(\int_{0}^{T} \int_{0}^{1} \frac{1}{2} \lambda^{2}\left(w_{x x}\right)^{2} d x d t\right)^{1 / 2} \\
& \approx\left(\sum_{n=0}^{T} \int_{0}^{1} \frac{1}{2} \lambda^{2}\left(\hat{w}_{x x}\right)^{2} d x \Delta t\right)^{1 / 2}
\end{aligned}
$$

with $\hat{w}$ the finite element approximation of the panel displacement. The relative error $\varepsilon_{U}$ in this norm is taken with respect to the reference solution $n_{e}=256$. The expected linear convergence in space and time is found, as both the spatial discretization and the time-marching scheme are first-order accurate.

\section{CONCLUSIONS}

The LF-TSD fluid equation is presented as a model that owes its popularity to its simplicity. This allows for relatively fast computations and easier interpretation of results where viscosity and vorticity effects are not dominant. A robust discretization scheme that resolves robustness issues encountered in $[5,6]$, is proposed. Conservation of the discretization is proven. The monolithic fluid-structure-interaction approach presented here does not rely on the definition of relaxation parameters. A series of 
numerical tests have displayed the performance of the discretization as well as the richness of the solution set of this relatively simple flow model.

(a)

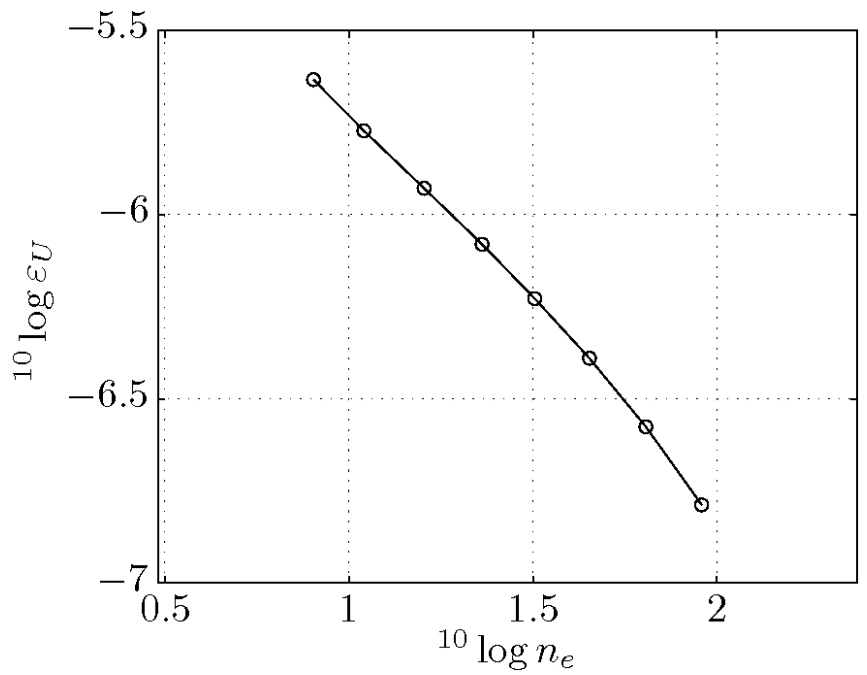

(b)

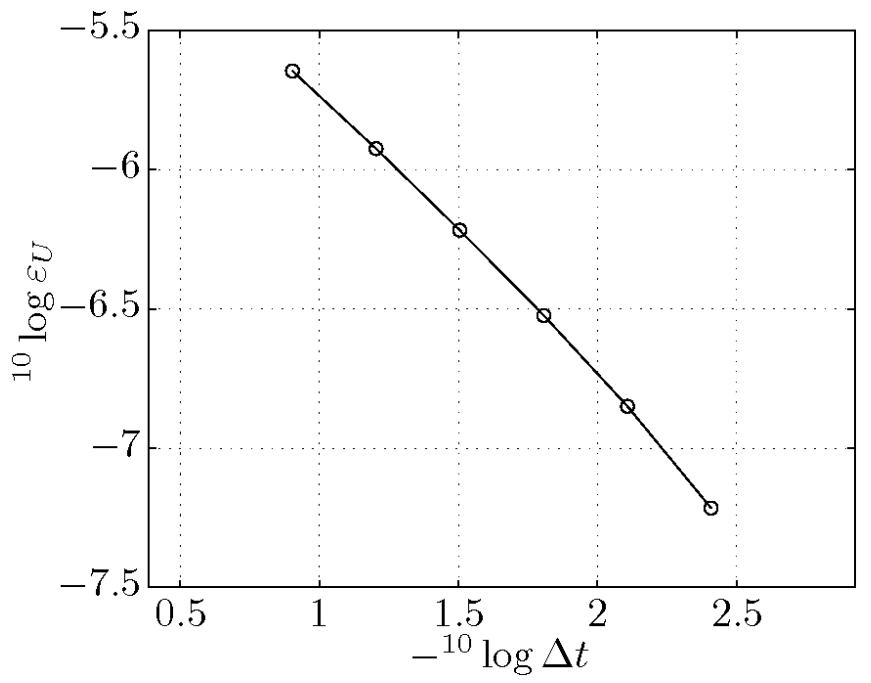

Fig. (9). Convergence plots in $\Delta x$ and $\Delta t$ for the LCO case.

\section{APPENDIX A}

\section{Conservation}

Here mass conservation is proven for the LF-TSD equation. Starting point is the divergence theorem, with $\nabla:=\{\partial / \partial t, \partial / \partial x, \partial / \partial y\}$ and $n=\left\{n_{t}, n_{x}, n_{y}\right\}^{T}$ :

$$
\begin{gathered}
\iiint_{\Omega \times] 0 ; T[} \nabla \cdot\{U, P, Q\} d t d x d y= \\
\oiint_{\substack{\partial \times] 0 ; T[ \\
U \Omega \times\{0, T\}}}\{U, P, Q\}^{T} \cdot \mathrm{n} d A .
\end{gathered}
$$

This integral can be approximated by dividing the domain $\Omega \times] 0 ; T$ [ into rectangular prism volumes with sides passing through the half-points defined in the paper. The discrete form of the divergence theorem then becomes:
$\left.\sum_{n=1}^{N} \sum_{i=1}^{H} \sum_{j=1}^{V}\left(U_{t}+P_{x}+Q_{y}\right)\right|_{i, j} ^{n} \Delta t \Delta x^{i} \Delta y^{j}=$

$\left.\sum_{i=1}^{H} \sum_{j=1}^{V} U_{i, j}^{n}\right|_{n=0} ^{N} \Delta x^{i} \Delta y^{j}+$

$\left.\sum_{n=1}^{N} \sum_{j=1}^{V} P_{i, j}^{n}\right|_{i=1} ^{H} \Delta t \Delta y^{j}+$

$\left.\sum_{n=1}^{N} \sum_{i=1}^{H} Q_{i, j}^{n}\right|_{j=1} ^{V} \Delta t \Delta x^{i}$

Integration is performed over $\Delta x^{i}:={ }_{1}^{1}{ }_{2}\left(\Delta x_{i-1}+\Delta x_{i}\right)$ and $\Delta y^{j}:=1 /{ }_{2}\left(\Delta y_{j-1}+\Delta y_{j}\right)$. For the factor $U_{t}+P_{x}+\left.Q_{y}\right|_{i, j} ^{n}$ the conservative form of the discrete LF-TSD equation (20) with (22) is substituted. Now write

$$
\begin{aligned}
& \forall i, j:\left.\sum_{n=1}^{N} U_{t}\right|_{i, j} ^{n} \Delta t \Delta x^{i} \Delta y^{j} \\
&=\left[U_{i, j}^{1}-U_{i, j}^{0}+\right. \\
&+U_{i, j}^{2}-U_{i, j}^{1}+ \\
&+ U_{i, j}^{3}-U_{i, j}^{2}+\ldots+ \\
&\left.+U_{i, j}^{N}-U_{i, j}^{N-1}\right] \Delta x^{i} \Delta y^{j} \\
&=\left.U_{i, j}^{N}-U_{i, j}^{0}\right] \Delta x^{i} \Delta y^{j} \\
& \forall n, j: \sum_{i=1}^{H}= P_{i, j}^{n} \Delta t \Delta x^{i} \Delta y^{j} \\
&=[\left(1-\mu_{1, j}\right)\left(P_{3 / 2, j}^{n}-P_{1 / 2, j}^{n}\right)+ \\
&+\mu_{0, j}\left(P_{1 / 2, j}^{n}-P_{-1 / 2, j}^{n}\right)+ \\
&+\left(1-\mu_{2, j}\right)\left(P_{5 / 2, j}^{n}-P_{3 / 2, j}^{n}\right)+ \\
&+\mu_{1, j}\left(P_{3 / 2, j}^{n}-P_{1 / 2, j}^{n}\right)+ \\
&+\left(1-\mu_{3, j}\right)\left(P_{7 / 2, j}^{n}-P_{5 / 2, j}^{n}\right)+ \\
&+\mu_{2, j}\left(P_{5 / 2, j}^{n}-P_{3 / 2, j}^{n}\right)+\ldots+ \\
&+\left(1-\mu_{H, j}\right)\left(P_{H+1 / 2, j}^{n}-P_{H-1 / 2, j}^{n}\right)+ \\
&\left.+\mu_{H-1, j}\left(P_{H-1 / 2, j}^{n}-P_{H-3 / 2, j}^{n}\right)\right] \Delta t \Delta y^{j} \\
&= {\left[\left(1-\mu_{H, j}\right) P_{H+1 / 2, j}^{n}+\mu_{H, j} P_{H-1 / 2, j}^{n}\right)+} \\
&\left.-\left(1-\mu_{0, j}\right) P_{1 / 2, j}^{n}-\mu_{0, j} P_{-1 / 2, j}^{n}\right] \Delta t \Delta y^{j}
\end{aligned}
$$

and

$$
\begin{aligned}
& \forall n, i:\left.\sum_{j=1}^{V} \quad Q_{y}\right|_{i, j} ^{n} \Delta t \Delta x^{i} \Delta y^{j} \\
& =\left[\begin{array}{ll}
\left(Q_{i, 3 / 2}^{n}-Q_{i, 1 / 2}^{n}\right.
\end{array}\right)+ \\
& +\left(Q_{i, 5 / 2}^{n}-Q_{i, 3 / 2}^{n}\right)+ \\
& +\left(Q_{i, 7 / 2}^{n}-Q_{i, 5 / 2}^{n}\right)+\ldots+ \\
& \left.+\left(Q_{i, V+1 / 2}^{n}-Q_{i, V-1 / 2}^{n}\right)\right] \Delta t \Delta x^{i} \\
& =\left[\begin{array}{ll}
Q_{i, V+1 / 2}^{n} & -Q_{i, 1 / 2}^{n}
\end{array}\right] \Delta t \Delta x^{i}
\end{aligned}
$$


where quantities $U, P$, and $Q$ lying outside the discrete domain $\Omega_{h}$ can be evaluated using the boundary conditions. Summing equation (30)-(32) over the remaining indices yields the required (29), conservation of the LF-TSD discretization in both space and time has thus been proven.

\section{APPENDIX B}

\section{Matrix Diagonals}

The diagonals in equations 16 are defined by:

$$
\begin{aligned}
& { }^{0} M_{k}=-1 \\
& { }^{-1} C_{k}=\frac{4 M_{\infty}}{\Delta x_{i-1}+\Delta x_{i}} \\
& { }^{0} C_{k}=\frac{-4 M_{\infty}}{\Delta x_{i-1}+\Delta x_{i}} \\
& { }^{-H} K_{k}^{\overline{n+1}}=\frac{2}{\Delta y_{j-1}\left(\Delta y_{j}+\Delta y_{j-1}\right)} \\
& { }^{-2} K_{k}^{\overline{n+1}}=2 \mu_{i-1, j}^{\overline{n+1}} \frac{A_{i-3 / 2, j}^{\overline{n+1}}}{\Delta x_{i-2}\left(\Delta x_{i-1}+\Delta x_{i}\right)} \\
& { }^{-1} K_{k}^{\overline{n+1}}=2\left(1-\mu_{i, j}^{\overline{n+1}}\right) \frac{A_{i-1 / 2, j}^{\overline{n+1}}}{\Delta x_{i-1}\left(\Delta x_{i-1}+\Delta x_{i}\right)}+ \\
& -2 \mu_{i-1, j}^{\overline{n+1}} \frac{A_{i-1 / 2, j}^{\overline{n+1}}}{\Delta x_{i-1}\left(\Delta x_{i-1}+\Delta x_{i}\right)}+ \\
& -2 \mu_{i-1, j}^{\overline{n+1}} \frac{A_{i-3 / 2, j}^{\overline{n+1}}}{\Delta x_{i-2}\left(\Delta x_{i-1}+\Delta x_{i}\right)} \\
& { }^{0} K_{k}^{\overline{n+1}}=-\sum_{m \in M \backslash\{0\}}{ }^{m} K_{k}^{\overline{n+1}} \\
& { }^{1} K_{k}^{\overline{n+1}}=2\left(1-\mu_{i, j}^{\overline{n+1}}\right) \frac{A_{i+1 / 2, j}^{\overline{n+1}}}{\Delta x_{i}\left(\Delta x_{i-1}+\Delta x_{i}\right)} \\
& { }^{H} K_{k}^{\overline{n+1}}=\frac{2}{\Delta y_{j}\left(\Delta y_{j}+\Delta y_{j-1}\right)} \\
& r_{k}^{\overline{n+1}}=-\frac{(\gamma+1) M_{\infty}^{2}}{\Delta x_{i-1}+\Delta x_{i}} \times \\
& \left\{\left(1-\mu_{i, j}^{\overline{n+1}}\right)\left[\left(\frac{\varphi_{i+1, j}^{\overline{n+1}}-\varphi_{i, j}^{\overline{n+1}}}{\Delta x_{i}}\right)^{2}-\left(\frac{\varphi_{i, j}^{\overline{n+1}}-\varphi_{i-1, j}^{\overline{n+1}}}{\Delta x_{i-1}}\right)^{2}\right]+\right. \\
& \left.\mu_{i-1, j}^{\overline{n+1}}\left[\left(\frac{\varphi_{i, j}^{\overline{n+1}}-\varphi_{i-1, j}^{\overline{n+1}}}{\Delta x_{i-1}}\right)^{2}-\left(\frac{\varphi_{i-1, j}^{\overline{n+1}}-\varphi_{i-2, j}^{\overline{n+1}}}{\Delta x_{i-2}}\right)^{2}\right]\right\} .
\end{aligned}
$$

\section{REFERENCES}

[1] O. Axelsson. Encyclopedia of Computational Mechanics, volume 1 Fundamentals. John Wiley \& sons, 1td., Chichester, 2004.

[2] W.F. Ballhaus and P.M. Goorjian. Implicit Finite-Difference Computations Of Unsteady Transonic Flows About Airfoils. AIAA Journal, vol. 15, no. 12, pp. 1728-1735, 1977.

[3] W.F. Ballhaus and P.M. Goorjian. Computation Of Unsteady Transonic Flows By The Indicial Method. AIAA Journal, Vol. 16, no. 2, pp. 117124, 1978.

[4] W.F. Ballhaus and H. Lomax. The numerical simulation of low frequency unsteady transonic flow fields. Proceedings of the fourth international conference on numerical methods in fluid dynamics. 1975, pp. 57-63.

[5] J.T. Batina. Efficient algorithm for solution of the unsteady transonic small-disturbance equation. Journal of Aircraft, vol. 25, no. 5, pp. 598605, 1988.

[6] C.J. Borland and D.P. Rizzetta. Nonlinear transonic flutter analysis AIAA Journal, vol. 20, no. 11, pp. 1606-1615, 1981.

[7] J. Chattot. Computational Aerodynamics and Fluid Dynamics. Springer-Verlag, Berlin / Heidelberg / New York, 2002.

[8] J.D. Cole and L.P. Cook. Transonic Aerodynamics. Amsterdam, NorthHolland, 1986.

[9] M. Giles. Non-Reflecting Boundary Conditions For The Euler Equations. Technical Report, CFDL-TR-88-1, MIT, Department Of Aeronautics And Astronautics, 1988.

[10] J.D. Anderson Jr. Fundamentals of Aerodynamics. McGraw-Hill: London, 2007.

[11] A. Kornecki and E.H. Dowell and J. O'Brien. Aeroelastic instability of 2-dimensional panels in uniform incompressible flow. Journal of Sound and Vibration, vol. 47, no. 2, pp. 163-178, 1976.

[12] E.M. Murman. Analysis Of Embedded Shock Waves Calculated By Relaxation Methods. AIAA Journal, vol. 12, no. 5, pp. 626-633, 1973.

[13] E.M. Murman and J.D. Cole. Calculation of plane steady transonic flows. AIAA Journal, vol. 9, no. 1, pp. 114-121, 1971

[14] S. Piperno and C. Farhat. Partitioned Procedures For The Transient Solution Of Coupled Aeroelastic Problem - Part II: Energy Transfer Analysis And Three-Dimensional Applications. Computer Methods in Applied Mechanics and Engineering, vol. 190, pp. 3147-3170, 2001.

[15] L.N. Sankar and M.J. Smith. Advanced transonic flow. Lecture notes, Georgia institute of technology, 1995.

[16] E.H. van Brummelen and K.G. van der Zee and R. de Borst. Space/time multigrid for a fluid--structure-interaction problem. Applied Numerical Mathematics, vol. 58, pp. 1951-1971, 2008.

[17] C.V. Verhoosel and T.P. Scholcz and S.J. Hulshoff and M.A. Gutiérrez. Uncertainty and reliability analysis of fluid-structure stability boundaries. AIAA Journal, vol. 47, no. 1, pp. 91-103, 2009.

[18] F. Verhulst. Nonlinear Differential Equations and Dynamical Systems. $2^{\text {nd }}$ Ed, Springer-Verlag, Berlin / Heidelberg / New York, 2006. 